\title{
Efficient Removal of Toxic Textile Dyes using Silver Nanocomposites
}

\author{
Khatoon $\mathbf{N}$ and Sardar $\mathbf{M}^{\star}$ \\ Department of Biosciences, Jamia Millia Islamia, New Delhi, India
}

*Corresponding author: Meryam Sardar, Department of Biosciences, Jamia Millia Islamia, New Delhi-110025, India, Tel: 011-26981717; E-mail: msardar@jmi.ac.in

Received date: August 22. 2017; Accepted date: September 08, 2017; Published date: September 15, 2017

Copyright: ( 2017 Khatoon N, et al. This is an open-access article distributed under the terms of the Creative Commons Attribution License, which permits unrestricted use, distribution, and reproduction in any medium, provided the original author and source are credited.

\begin{abstract}
In the present study biomimetic synthesis of silver nanoparticles using Solanum nigrum and Cannabis sativa were explored. The biosynthesized silver nanocomposites were characterized using UV-VIS spectroscopy, DLS, TEM, FTIR and XRD. The characteristic surface plasmon resonance of Solanum nigrum and Cannabis sativa were recorded at 430 and $445 \mathrm{~nm}$ respectively. The average diameter of silver nanocomposites were $34.13 \pm 3.10$ and $70.93 \pm 3.57 \mathrm{~nm}$ for Solanum nigrum and Cannabis sativa leaf extract respectively. The release of textile dyes into our surrounding water bodies has toxic effect on human health and marine life therefore removal of these dyes is necessary to protect environment. The nanotechnology has provided a new platform for waste water treatment. Hence the biosynthesized nanocomposites were employed for dye removal from wastewater. The effects of various parameters, such as time of incubation, concentration, $\mathrm{pH}$, and temperature, were studied. The biosynthesized silver nanoparticles are most effective in dye removal at alkaline $\mathrm{pH} 9$ and at $60^{\circ} \mathrm{C}$. The textile industry effluents have high $\mathrm{pH}$ and temperature which makes these nanoparticles more appropriate in treatment of these industry effluents. To elucidate the reusability, the decolorizing efficiency of biosynthesized silver nanocomposites were investigated upto 3 cycles.
\end{abstract}

Keywords Green nanoparticles; Textile dye removal; Catalytic; Biosynthesized

\section{Introduction}

Nanotechnology is the science for tailoring and manufacturing macro atoms into nano materials. Inorganic nano materials are gaining attention due to their novel and size-related physico-chemical properties as compared to bulk matter [1]. Due to their these novel properties of nano materials, they have been used in a wide range of potential applications in medicine, cosmetics, catalyst, renewable energy and biomedical devices [2-6].

Among nano materials, silver nano materials have been focused due to their distinctive physical, chemical and biological properties as compared to macroscopic materials [7]. Silver nanoparticles have distinctive physico-chemical properties, such as high electrical and thermal conductivity, chemical stability, catalytic activity and non linear optical behaviour [4,8-10]. Besides this silver nanoparticles have also been used as antimicrobial agents, biosensors and waste water purification etc. [11-13]. Currently, different methods are known for the synthesis of silver nanoparticles by using chemical, physical and biological routes [14].

The chemical methods are the most popular but the use of toxic chemicals during synthesis produces toxic by-products [15]. The physical methods required ample amount of energy to maintain high pressure and temperature. Although chemical and physical methods may successfully produce pure well-defined nanomaterials, these are also cost oriented and not good for sustainable ecosystem [15]. To overcome the disadvantage of chemical and physical methods, the researchers have migrated to biological systems which utilize ecofriendly technique for the synthesis of nanoparticles [16]. Green nanotechnology is an alternative to the conventional physicochemical approaches for the synthesis of nanomaterials in a clean, benign and eco friendly environment [16].

Various industries, for example, plastics, paper, textile and dyestuffs, devour considerable volume of water, and utilize chemicals and dyes in manufacturing which generates substantial amount of waste water $[17,18]$. One of the essential contaminant to be perceived in waste water is color which are highly visible and undesirable even in very low concentrations of dyes [19].

It is very well known that dyes enriched wastewaters are extremely hard to treat, since the dyes are recalcitrant molecules (particularly azo dyes), stable to oxidizing agents and resistant to aerobic digestion. The release of dyes into our surrounding water bodies has toxic effect on human health and marine life [20].

Therefore removal of these dyes is necessary to protect environment. The nanotechnology has been provided a new platform for waste water treatment [21]. The researchers have explored the catalytic and dye degradation of silver nanoparticles [22]. So in the present study green synthesized silver nanoparticles are explored for its efficiency in removing the textile dyes.

\section{Materials and Methods}

\section{Materials}

Fresh Solanum nigrum and Cannabis sativa leaves were collected from Jamia Millia Islamia campus in the month of January. Silver nitrate, ethanol were procured from Hi- Media.

\section{Synthesis and purification of silver nanocomposites using leaf extract}

Fresh leaves of Solanum nigrum (10 gm) and Cannabis sativa (15 $\mathrm{gm})$ were taken and washed thoroughly to remove dust particles. After 
air drying, chopped leaves were mixed to $100 \mathrm{ml} 50 \%$ ethanol and were kept at $60^{\circ} \mathrm{C}$ for $10-15$ minutes till the color of the solvent changes to light green. The mixture was cooled and filtered through Whatmann filter paper. This filtrate was used as precursor and was utilized in subsequent procedures for biosynthesis. For each plant synthesis of silver nanocomposites was carried out by mixing of leaf extract with freshly prepared aqueous solution of $\mathrm{AgNO}_{3}(2 \mathrm{mM})$ in 1:9 ratio. Purification was done as described earlier in our lab [23].

\section{UV-VIS spectroscopy}

The bioreduction of Ag+ ions by leaf extract will be monitored by measuring the UV-Vis spectra of the reaction medium. UV-VIS spectral studies were done by using a Mecasys Optizen 3220UV spectrophotometer. The experiments were performed thrice.

\section{Dynamic light scattering}

DLS measurements for determining the average size and size distribution of the silver nanoparticles were carried out using the spectroscatterer RiNA, GmbH class3B. The dried powder was dispersed in distilled water and all the analysis were done at $20^{\circ} \mathrm{C}$ for 10 cycles. Experiments for DLS were repeated thrice.

\section{TEM measurements}

TEM measurement of was performed on a JEOL model JEM-2000FX instrument operated at an accelerating voltage of $200 \mathrm{kV}$. TEM samples were prepared by placing a drop of sonicated powdered sample, in absolute ethanol for about $15 \mathrm{~min}$ on ultrasonicator (UP-500 Ultrasonic Processor), on a carbon coated copper grid and dried in air for $1 \mathrm{~h}$.

\section{Fourier transform infrared (FTIR)}

Spectra of washed and purified silver nanocomposites were recorded on a Perkin Elmer FTIR spectroscopy using $\mathrm{KBr}$ pellets. To obtain good signal to noise ratio, 32 scans of nanosilver were taken in the range $400-4000 \mathrm{~cm}^{-1}$ and the resolution was kept as $4.0 \mathrm{~cm}^{-1}$.

\section{Removal of dyes by silver nanocomposites}

Two local dyes used in textile industry (Yellow and blue) were collected from local vendors of Punjabi Bagh, New Delhi and 10 $\mathrm{mg} / \mathrm{ml}$ of dyes were dissolved in distilled water. Parameters optimized included concentration of silver nanocomposites $(1-4 \mu \mathrm{g} / \mathrm{mL}), \mathrm{pH}(4.0$ to 11.0$)$ and temperature $\left(40,50,60,70\right.$ and $\left.80^{\circ} \mathrm{C}\right)$ at constant speed of agitation $(180 \mathrm{rpm})$. The percent decolourization was calculated by taking untreated dye solution as control (100\%).

\section{Statistical analysis}

ANOVA was performed followed by Dunnets test and values were represented as means of three replicate $(n=3) \pm S D$. The significance level was maintained as $\mathrm{p}$ - value $<0.05$.

\section{Results and Discussion}

In the present study two different plant assisted silver nanocomposites were analysed for removal of textile dyes. Although several reports have been published for the synthesis of silver nanocomposites using plants/plant extract, till date Solanum nigrum and Cannabis sativa plants have not been explored for biosynthesis of silver nanocomposites. Solanum nigrum is also known as Black Nightshade or Makoi is a perennial shrub and grows as a weed with agricultural crops, also considered an important shrub in Ayurveda.

Cannabis sativa is also annually herbaceous plant commonly known as marijuana, due to its psychotropic properties its is used in treatment of various ailments. The easy cultivation and availability throughout the year makes these medicinal plants a suitable candidates for synthesis of silver nanoparticles. The colorless solution of silver nitrate turns to dark brown due to the synthesis of silver nanocomposites. The surface plasmon vibrations are responsible for color change of solution.

The peak for surface plasmon resonance were recorded at UV-Vis spectrophotometer. The $\lambda \max$ for Solanum nigrum and Cannabis sativa leaf extract were at $430 \mathrm{~nm}$ and $445 \mathrm{~nm}$ respectively. The critical parameters such as time, temperature, concentration of leaf extract and silver nitrate which dictates the synthesis were optimized.

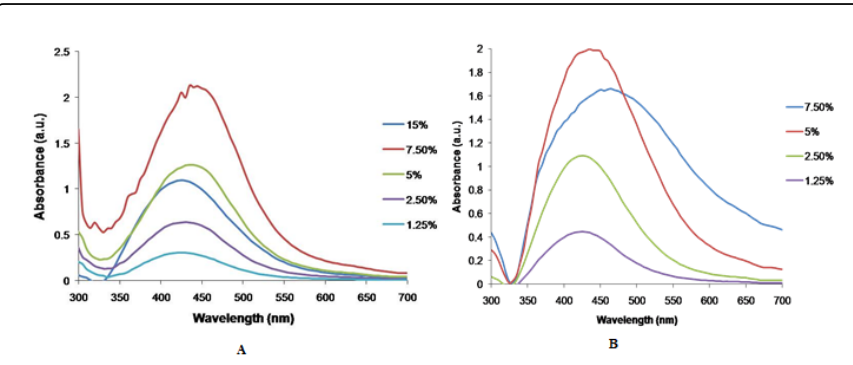

Figure 1: UV-Vis spectra recorded as a function of concentration of Solanum nigrum 1A) and Cannabis sativa 1B) leaf extract for the synthesis of silver nanocomposites. Spectrophotometer were operated at a resolution of $1 \mathrm{~nm}$.

The Figure 1 shows the effect of concentration of leaf extract and the best results were obtained at concentration of $7.5 \%$ for Solanum nigrum (Figure 1A) and for Cannabis sativa was 5\% (Figure 1B).

Further the concentration of silver nitrate was varied. The results shows that as the concentration of silver nitrate increases the absorption intensity increases upto $2 \mathrm{mM}$ for both the nanocomposites, beyond that intensity started decreasing (Figures $2 \mathrm{~A}$ and $2 \mathrm{~B}$ ). To study the effect of time on synthesis, the UV-Vis spectrum of the solution were recorded at different time period (Figures $3 \mathrm{~A}$ and $3 \mathrm{~B})$.

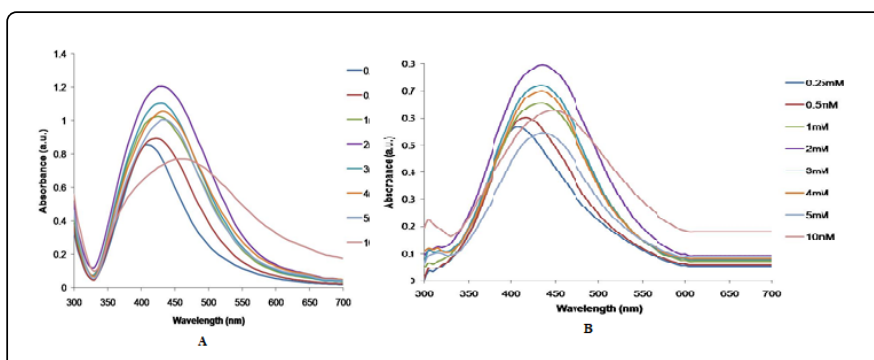

Figure 2: UV-Vis spectra recorded as a function of concentration of silver nitrate for the synthesis of silver nanocomposites assisted by 2A) Solanum nigrum and 2B) Cannabis sativa leaf extract. Spectrophotometer were operated at a resolution of $1 \mathrm{~nm}$. 

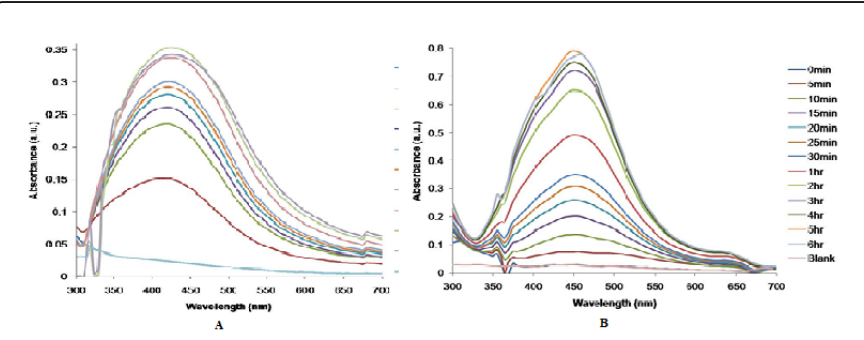

Figure 3: UV-Vis spectra recorded as a function of time for the synthesis of silver nanocomposites assisted by 3 A) Solanum nigrum and 3B) Cannabis sativa leaf extract. Spectrophotometer were operated at a resolution of $1 \mathrm{~nm}$.

The results show that absorption increases up to $2 \mathrm{~h}$ for Solanum nigrum and $5 \mathrm{hr}$ for Cannabis sativa, after that it is stable. Effect of incubation time has a significant role in synthesis this has been reported earlier also [24]. The controls of only Solanum nigrum and Cannabis sativa leaf extract and silver nitrate did not show any color and consequently did not have any peak in the visible region.

To determine the hydrodynamic radius of biosynthesized silver nanopartic the DLS were carried out. The DLS pattern reveals that silver nanocomposites synthesized by this method have average diameter of $34.13 \pm 3.10$ and $70.93 \pm 3.57 \mathrm{~nm}$ for Solanum nigrum (Figure 4A) and Cannabis sativa (Figure 4B) leaf extract respectively.

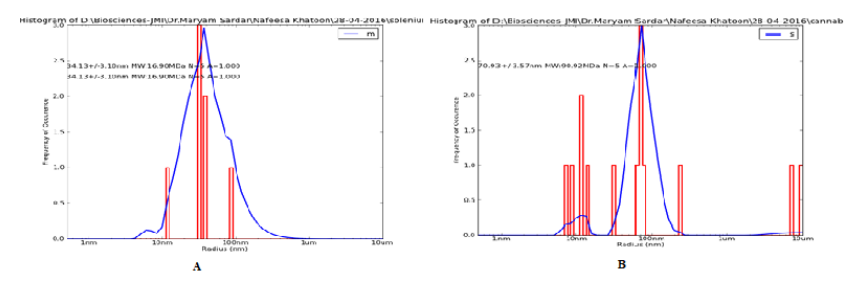

Figure 4: DLS of biosynthesized silver nanocomposites assisted by 4A) Solanum nigrum and 4B) Cannabis sativa leaf extract. Aqueous solution of silver nanoparticles $(1 \mathrm{mg} / \mathrm{ml})$ was prepared and all the analysis were done at $20^{\circ} \mathrm{C}$ for 10 cycles.

Polydispersity index (PDI) were determined to know the particle size distribution of these biosynthesized silver nanoparticles and PDI was found to be 0.28 for Solanum nigrum assisted silver nanoparticles and 0.33 Cannabis sativa assisted silver nanoparticles. The stability of colloidal solutions were determined by zeta potentials and were found to be +39.75 and +34.25 for Solanum nigrum and Cannabis sativa assisted silver nanoparticles.

These values of zeta potential indicate a long-term stability of the corresponding colloids, which may be due to biomolecules responsible for stabilization of nanoparticles. The size and shape of silver nanocomposites were also determined using TEM.

The TEM data reveals that biosynthesized silver nanocomposites are spherical in shape having 5-17 nm for Solanum nigrum (Figure 5A) and 12-22 nm for Cannabis sativa (Figure 5B).

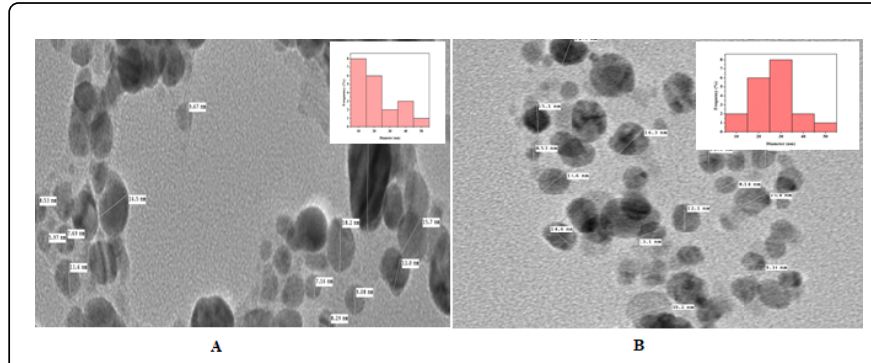

Figure 5: TEM of biosynthesized silver nanocomposites assisted by 5A) Solanum nigrum and 5B) Cannabis sativa leaf extract. The samples were prepared in absolute ethanol by placing a drop on a carbon coated copper grid.

The biomolecules responsible for reduction and stabilization of silver nanocomposites were studied using FTIR. The peaks for Solanum nigrum assisted nanocomposites were at 3482, 1631 and for only Solanum nigrum leaf extract were at 3482, 1631and 1042. The peak 3482 corresponds to -OH, 1631 for amide I and $1042 \mathrm{C}-\mathrm{H}$ bond rotation (Figure 6A). The prominent peaks for Cannabis sativa assisted nanocomposites were at $3424(-\mathrm{OH})$ and $1644(\mathrm{C}=\mathrm{O})$. The peaks for only Cannabis sativa leaf extract were $3424(-\mathrm{OH}), 2988(\mathrm{C}-\mathrm{H}), 1644$ $(\mathrm{C}=\mathrm{O}), 1047(\mathrm{C}-\mathrm{O})$ and $847(\mathrm{C}-\mathrm{O}-\mathrm{C})$ (Figure 6B). In all FTIR spectra the $-\mathrm{OH}$ peak is most abundant due to the wide presence of the- $\mathrm{OH}$ functional group in tannins, flavonoids and eugenol [25]. The other peaks are indicating the presence of organic moieties on the metal surface of particles [25].
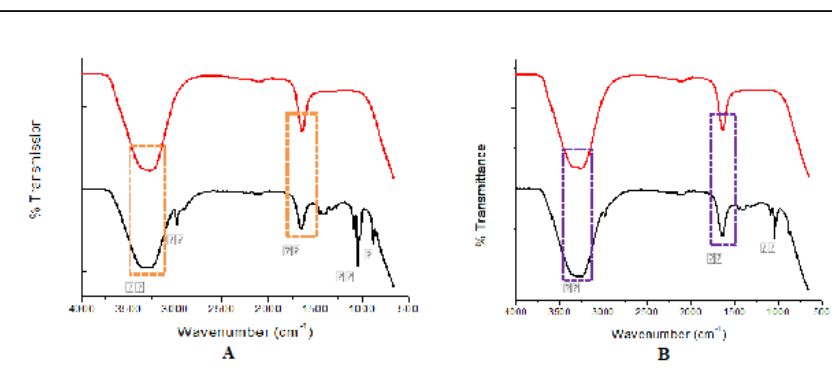

Figure 6: FTIR of Solanum nigrum and Cannabis sativa assisted silver nanocomposites where represents $6 \mathrm{~A}$ ) silver nanocomposites and $6 \mathrm{~B}$ ) leaf extract.

The silver nanocomposites were known for its catalytic properties. Therefore the biosynthesized silver nanocomposites were exploited for the hazardous and toxic textile dye removal. The percentage of dye removal known to have been influenced by various parameters such as concentration of silver nanocomposites, time and temperature [13]. The concentration of silver nanocomposites are considered to be a vital factor, therefore effect of concentration of silver nanocomposites were studied. As the concentration of silver nanocomposites increases from 1 to $4 \mu \mathrm{g} / \mathrm{ml}$, the efficiency of dye removal increases upto $3 \mu \mathrm{g} / \mathrm{ml}$ and after this the dye removal efficiency becomes constant (Figure 7). 


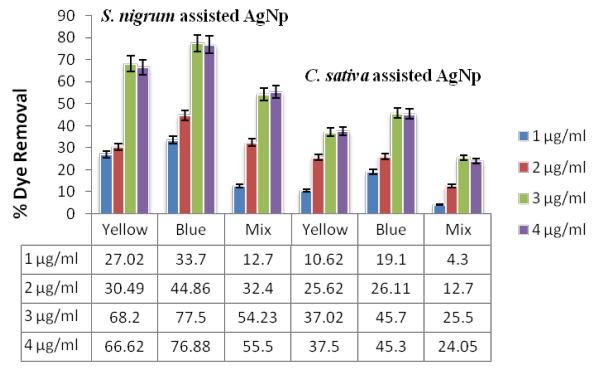

Figure 7: Effect of concentration Solanum nigrum and Cannabis sativa assisted silver nanocomposites on dye removal. All the values are means of triplicate $(\mathrm{n}=3) \pm S \mathrm{SD}$. ANOVA significant at $\mathrm{P} \leq 0.05$.

This may be due to achievement of equilibirium as a given amount of adsorbed has the capacity to adsorb fixed amount of adsorbent and after equilibirum it becomes constant. The Solanum nigrum mediated silver nanocomposites at $3 \mu \mathrm{g} / \mathrm{ml}$ for yellow, blue and mixture of both the dyes are $68.2 \%, 77.5 \%$ and $54.23 \%$ respectively while Cannabis sativa mediated silver nanocomposites at same concentration are $27.02 \%, 45.7 \%$ and $25.5 \%$.

The effect of $\mathrm{pH}$ on dye removal was also studied, for this, the $\mathrm{pH}$ of the solution was adjusted to $4,7,9,10$ and 11 and $2 \mu \mathrm{g} / \mathrm{ml}$ silver nanocomposites were added to each solution and all were incubated at $60^{\circ} \mathrm{C}$ for $2 \mathrm{hrs}$ with constant agitation and the results obtained are shown in Figure 8. The maximum dye removal (yellow, blue and mixture) were obtained at basic $\mathrm{pH}$ that is at $\mathrm{pH} 9$ for both the nanocomposites. This may be due to electrostatic interaction of dyes with nanocomposites which varies with the change in $\mathrm{pH}$ [13].

Hence, the alkaline $\mathrm{pH}$ was maintained for all other experiments. Another important parameter that taken into account is temperature which greatly influenced potential of dye removal and therefore batch experiments were carried out at different temperatures. The temperature of the solution was varied from $40-80^{\circ} \mathrm{C}$ with constant agitation.

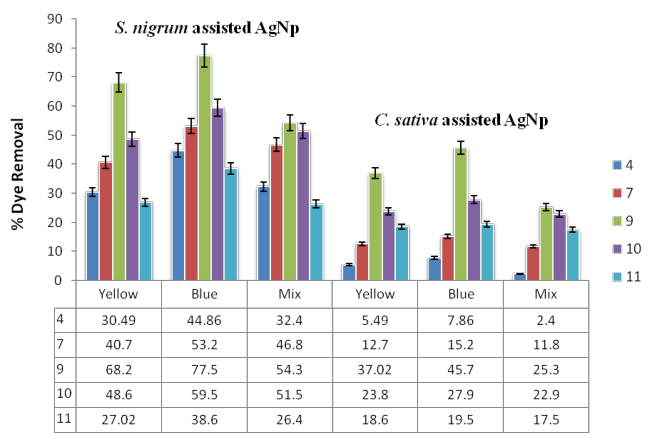

Figure 8: Effect of $\mathrm{pH}$ of Solanum nigrum and Cannabis sativa assisted silver nanocomposites on dye removal. All the values are means of triplicate $(n=3) \pm S D$. ANOVA significant at $P \leq 0.05$.

Figure 9 reveals that by keeping all other conditions constant, an increase in temperature led to an increase in the percentage of dye adsorption upto $60^{\circ} \mathrm{C}$ for Solanum nigrum and Cannabis sativa assisted silver nanocomposites respectively, after that there is decrease in efficiency. This might be explained by a probable increase in affinity of binding sites of the adsorbent for dye molecules with a corresponding increase in temperature [13]. Rise in temperature may also have resulted in a higher mobility of the dye molecules accompanied by a reduction in the retarding forces acting on the same thereby incrementing the adsorbent efficiency. The decrease of dye removal beyond $60^{\circ} \mathrm{C}$ is may be due to denaturation of biomolecules present on silver nanocomposites at higher temperature.

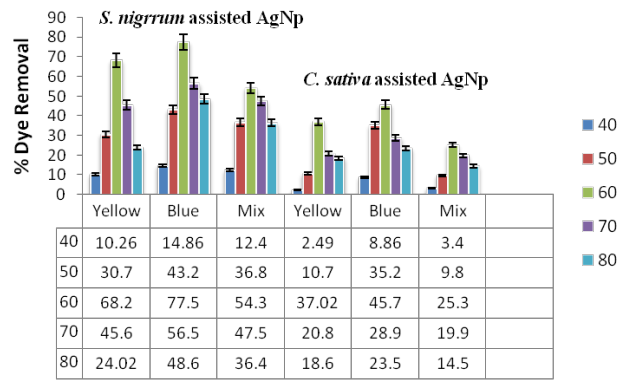

Figure 9: Effect of temperature of Solanum nigrum and Cannabis sativa assisted silver nanocomposites on dye removal. All the values are means of triplicate $(n=3) \pm S D$. ANOVA significant at $\mathrm{P} \leq 0.05$.

Figure 10 shows the visual observations of dye removal when incubated with Solanum nigrum and Cannabis sativa assisted silver nanocomposites at a concentration of $3 \mu \mathrm{g} / \mathrm{ml}, \mathrm{pH} 9$ at $60^{\circ} \mathrm{C}$ for $2 \mathrm{hrs}$. The biosynthesized nanocomposites were studied for their reusability and the results shows that they can be reused upto 3 cycles efficiently. The study indicates that Solanum nigrum assisted silver nanocomposites are better in dye removal as compared to Cannabis sativa as the efficiency of dye removal for Solanum nigrum is two times more as compared to Cannabis sativa this may be due to small size of Solanum nigrum assisted silver nanocomposites. Solanum nigrum can be grown easily throughout the year thus it can be used for large scale synthesis of silver nanocomposites. These nanocomposites can be incorporated into membrane filters to enhance their efficiency.

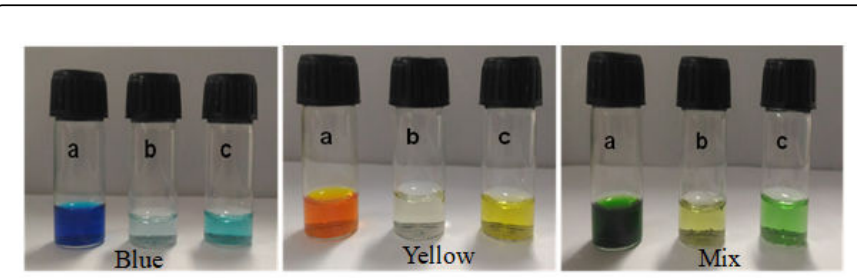

Figure 10: Visual observation of dye removal with and without silver nanocomposites where a corresponds to dyes without nanocomposites and b and c corresponds to dyes with Solanum nigrum and Cannabis sativa assisted silver nanocomposites.

\section{Conclusion}

Silver nanocomposites has been successfully prepared by green route using two medicinal plants leaf extract i.e., Solanum nigrum and Cannabis sativa. These biosynthesized silver nanocomposites were implemented for removal of textile dyes from its aqueous solution. The 
Reactive textile dyes are highly soluble in nature and the total dye is in not taken up by the textile fibres which result in $10-15 \%$ of dyes being discharged in wastewater. The study concludes that Solanum nigrum is more effective than Cannabis sativa in the reduction of water impurities from colored dyes. In totality, the study suggests achievement of an ecofriendly and highly efficient adsorbent which may be considered useful for the removal of dyes from textile industries.

\section{Acknowledgement}

The author acknowledge the UGC and Indian Council of Medical Research (grant number 35/8/2012-BMS), Govt. of India, for providing the financial support.

\section{References}

1. Peto G, Molnar GL, Paszti Z, Geszti O, Beck A, et al. (2002) Electronic structure of gold nanoparticles deposited on $\mathrm{SiO} x / \mathrm{Si}$ (100). Mater Sci Eng C: C 19: 95-9.

2. Premkumar T, Lee Y, Geckeler KE (2010) Macrocycles as a tool: A facile and one pot synthesis of silver nanoparticles using cucurbituril designed for cancer therapeutics. Chem Eur J 16: 11563-66.

3. Vidhu VK, Philip D (2014) Spectroscopic, microscopic and catalytic properties of silver nanoparticles synthesized using Saraca indica flower. Spectrochimica Acta Part A: Molecular and Biomolecular Spectroscopy 117: 102-8.

4. Xu R, Wang D, Zhang J, Li Y (2006) Shape dependent catalytic activity of silver nanoparticles for the oxidation of styrene. Chem Asian J 1: 888-93.

5. Somorjai GA, Frei H, Park JY (2009) Advancing the frontiers in nanocatalysis, biointerfaces, and renewable energy conversion by innovations of surface techniques. J Am Chem Soc 131: 16589-605.

6. Furno F, Morley KS, Wong B, Sharp BL, Arnold PL, et al. (2004) Silver nanoparticles and polymeric medical devices: A new approach to prevention of infection? J Antimicrob Chemother 54: 1019-24.

7. Dwivedi AD, Gopal K (2010) Biosynthesis of silver and gold nanoparticles using Chenopodium album leaf extract. Colloids and Surfaces A: Physicochemical and Engineering Aspects 369: 27-33.

8. Jain PK, Huang X, El-Sayed IH, El-Sayed MA (2008) Noble metals on the nanoscale: optical and photothermal properties and some applications in imaging, sensing, biology, and medicine. Acc Chem Res 41: 1578-86.

9. Mafune F, Kohno JY, Takeda Y, Kondow T, Sawabe H (2000) Structure and stability of silver nanoparticles in aqueous solution produced by laser ablation. J Phys Chem B 104: 8333-37.

10. Tran QH, Le AT (2013) Silver nanoparticles: synthesis, properties, toxicology, applications and perspectives. Advances in Natural Sciences: Nanoscience and Nanotechnology $4: 3$.
11. Park Y (2014) New paradigm shift for the green synthesis of antibacterial silver nanoparticles utilizing plant extracts. Toxicol Res 30:169.

12. Lin J, He C, Zhao Y, Zhang S (2009) One-step synthesis of silver nanoparticles/carbon nanotubes/chitosan film and its application in glucose biosensor. Sens Actuators B Chem 137: 768-73.

13. Banerjee P, Sau S, Das P, Mukhopadhyay A (2014) Green synthesis of silver-nanocomposite for treatment of textile dye. Nanosci Technol 1: 1-6.

14. Huang Z, Jiang X, Guo D, Gu N (2011) Controllable synthesis and biomedical applications of silver nanomaterials. J Nanosci Nanotechnol 11: 9395-408.

15. Tripathy A, Raichur AM, Chandrasekaran N, Prathna TC, Mukherjee A (2010) Process variables in biomimetic synthesis of silver nanoparticles by aqueous extract of Azadirachta indica (Neem) leaves. J Nanopar Res 12: 237-46.

16. Arunachalam KD, Annamalai SK, Hari S (2013) One-step green synthesis and characterization of leaf extract-mediated biocompatible silver and gold nanoparticles from Memecylon umbellatum. Int J Nanomed 8: 1307.

17. Ali M, Sreekrishnan TR (2001) Aquatic toxicity from pulp and paper mill effluents: a review. Adv Environ Res 5: 175-96.

18. Robinson T, McMullan G, Marchant R, Nigam P (2001) Remediation of dyes in textile effluent: a critical review on current treatment technologies with a proposed alternative. Bioresour Technol 77: 247-55.

19. Banat IM, Nigam P, Singh D, Marchant R (1996) Microbial decolorization of textile-dyecontaining effluents: A review. Bioresour Technol 58: 217-27.

20. Khan S, Malik A (2014) Environmental and health effects of textile industry wastewater. Environmental Deterioration and Human Health, pp: $55-71$.

21. G Moussavi, M Mahmoudi (2009) Removal of azo and anthraquinone reactive dyes from industrial wastewaters using $\mathrm{MgO}$ nanoparticles. J Hazard Mater 168: 806-12.

22. Ai L, Jiang J (2013) Catalytic reduction of 4-nitrophenol by silver nanoparticles stabilized on environmentally benign macroscopic biopolymer hydrogel. Bioresour Technol 132: 374-77.

23. Khatoon N, Mishra A, Alam H, Manzoor N, Sardar M (2015) Biosynthesis, characterization, and antifungal activity of the silver nanoparticles against pathogenic Candida species. BioNanoScience 5: 65-74.

24. Jha AK, Prasad K (2010) Green synthesis of silver nanoparticles using Cycas leaf. Int J Green Nanotechnol: Physics and Chemistry 1: P110-17.

25. Parashar UK, Kumar V, Bera T, Saxena PS, Nath G, Srivastava SK, et al. (2011) Study of mechanism of enhanced antibacterial activity by green synthesis of silver nanoparticles. Nanotechnol 22: 415104. 